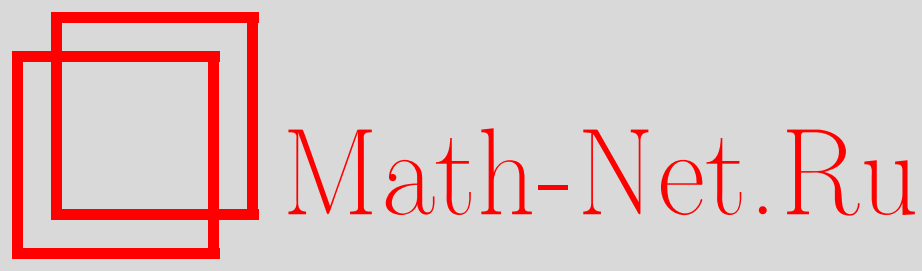

А. Н. Васильев, А. В. Кулиш, Распределение жидкости и эффективный потенциал в ограниченной системе специальной геометрии, ТМФ, 2010, том 163, номер 1, 156162

DOI: https://doi.org/10.4213/tmf6493

Использование Общероссийского математического портала Math-Net.Ru подразумевает, что вы прочитали и согласны с пользовательским соглашением http://www.mathnet.ru/rus/agreement

Параметры загрузки:

IP: 52.23 .180 .231

26 апреля 2023 г., 17:18:28

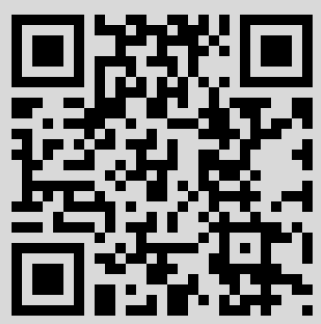




\title{
РАСПРЕДЕЛЕНИЕ ЖИДКОСТИ И ЭФФЕКТИВНЫЙ ПОТЕНЦИАЛ В ОГРАНИЧЕННОЙ СИСТЕМЕ СПЕЦИАЛЬНОЙ ГЕОМЕТРИИ
}

\begin{abstract}
Вычисляется плотность распределения жидкости, ограниченной системой коаксиальных цилиндров с заданным пристеночным потенциалом. Задача решается в рамках приближения плавной неоднородности для пристеночного потенциала произвольного типа. На основании полученного решения анализируется частный случай, когда пристеночный потенциал является существенно короткодействующим и в области действия остается постоянным внутри каждой из прослоек. Для этого случая вычисляется эффективный пристеночный потенциал, при котором распределение плотности является непрерывной функцией во всей области.
\end{abstract}

Ключевые слова: распределение плотности, пристеночный потенциал, пространственно ограниченная система.

\section{1. ВСТУПЛЕНИЕ}

В теории жидкостей важную информацию о структуре и свойствах системы получают на основе экспериментов по рассеянию нейтронов [1]-[3]. В частности, по интенсивности рассеяния нейтронов можно восстановить профиль плотности жидкости в образце, поскольку основным фактором, определяющим интенсивность рассеяния нейтронов, является характер распределения жидкости. Более того, эксперименты по рассеянию нейтронов являются базовыми в исследовании вопроса о зависимости распределения жидкости от температуры, особенно в критической области. Данная задача имеет прямое отношение к проблеме определения уравнения состояния жидкой системы и является сложной и, как известно, в общем случае не решенной. На сегодняшний день уровень экспериментальных методик и технологий позволяет достаточно точно и надежно определять профиль распределения плотности жидкости в образцах практически любой формы. Для обработки таких

\footnotetext{
${ }^{*}$ Киевский национальный университет им. Тараса Шевченко, Киев, Украина. E-mail: vasilev@univ.kiev.ua

${ }^{\dagger}$ Национальный технический университет Украины “КПИ”, Киев, Украина
} 
экспериментов необходима простая и продуктивная теория. Хотя система уравнений движения для жидкости, состоящая из уравнения непрерывности, уравнения Навье-Стокса и уравнения теплопроводности (с учетом переноса вещества), является замкнутой, она содержит, кроме скорости жидкости (которая в рассматриваемом далее случае равна нулю), давление как функцию плотности и температуры. Другими словами, чтобы решить указанную систему уравнений гидродинамики, необходимо знать уравнение состояния вещества (жидкости). К сожалению, это уравнение в общем случае неизвестно. Таким образом, для восстановления по данным нейтронного рассеяния уравнения состояния жидкости (хотя бы для ограниченного диапазона температур) необходимо сделать какие-то предположения относительно этого уравнения состояния. Учитывая данное обстоятельство и тот факт, что построение продуктивной теории жидких систем представляется задачей исключительно сложной, на практике прибегают к различного рода феноменологическим моделям, которые, с одной стороны, просты для выполнения аналитического прогнозирования свойств систем, а с другой, позволяют получать достоверные, согласующиеся с экспериментальными данными, результаты (см., например, [2], [4]).

Такой подход использован и в настоящей статье. В частности, в качестве отправной точки модели рассматривается феноменологическое выражение для добавки к свободной энергии, обусловленной неоднородностью распределения жидкости в образце и возникающей вследствие взаимодействия жидкости с ограничивающими систему поверхностями. Взаимодействие жидкости с поверхностями описывается пристеночным потенциалом (создаваемым поверхностями), который полагается известным. Задача о распределении плотности решается путем минимизации функционала с учетом дополнительного условия (неизменность общей массы жидкости в образце). Более конкретно, в настоящей статье вычисляется профиль распределения жидкости в пространственно ограниченной системе, состоящей из вложенных друг в друга коаксиальных цилиндров. Эта задача имеет прикладное значение в силу сразу нескольких причин. Во-первых, как отмечалось выше, наличие точного в рамках исходной модели решения позволяет рассчитать, при сравнении с экспериментом, ряд важных характеристик жидкой системы (таких, например, как интенсивность рассеяния нейтронов). Во-вторых, такого рода задачи актуальны с точки зрения более глобальной проблемы анализа заполнения малоразмерных систем разной геометрии жидким содержимым. Подобные проблемы возникли вместе с развитием нанотехнологий и связаны с тем, что заполнение нанопор жидким содержимым крайне усложняется из-за наличия поверхностного натяжения, которое препятствует проникновению жидкости внутрь поры. Один из способов разрешения этой технологической трудности состоит в том, что жидкость переводится в околокритическое состояние, в котором коэффициент поверхностного натяжения близок к нулю, после чего такой "почти критической” жидкостью заполняются поры.

Если речь идет о макросистемах упомянутой геометрии, когда стенки, разделяющие слои жидкости, достаточно толстые, то такая система может рассматриваться как простое, механическое объединение подсистем, ограниченных парой цилиндров соответствующих радиусов. Решение задачи о распределении жидкости в данной 
системе сводится к поиску решения для жидкости, ограниченной двумя цилиндрами, с заданной формой пристеночного потенциала. Более интересной является ситуация, когда стенки цилиндров тонкие - порядка нескольких атомарных слоев. В известном смысле такие стенки можно рассматривать как наносетку. Главная особенность такой системы состоит в том, что распределение плотности жидкости должно быть непрерывным в пределах всей системы, а не только отдельной прослойки. Данную ситуацию мы рассматриваем при вычислении эффективного пристеночного потенциала.

В нашей статье сначала решается вспомогательная задача о распределении плотности жидкости между двумя коаксиальными цилиндрами под действием внешнего поля (в качестве которого затем рассматривается пристеночный потенциал, т.е. потенциал взаимодействия между ограничивающими систему стенками и жидкостью). Задача решается в предположении, что профиль плотности между коаксиальными цилиндрами искривляется (по сравнению с однородным распределением) в силу действия поля. Поскольку точное выражение для функционала свободной энергии системы неизвестно, выполняется разложение этого функционала по отклонениям плотности от среднего значения и по градиенту до второго порядка включительно. Данный подход соответствует приближению плавной неоднородности [5], [6].

Следует отметить, что метод, использованный в настоящей статье, впервые был предложен в серии работ по исследованию однокомпонентных пространственно ограниченных систем простой геометрии (плоский слой, цилиндр и сфера), находящихся под действием гравитационного поля и пристеночного потенциала определенного вида [5], [6]. Впоследствии этот метод был расширен на общий случай внешнего потенциала в многокомпонентных пространственно ограниченных системах, находящихся под действием внешнего поля [7].

\section{2. ОГРАНИЧЕННАЯ СИСТЕМА С ГЕОМЕТРИЕЙ КОАКСИАЛЬНЫХ ЦИЛИНДРОВ}

Для начала рассмотрим вспомогательную задачу о распределении плотности однокомпонентной жидкости, ограниченной двумя коаксиальными цилиндрами радиусов $R_{1}$ и $R_{2}>R_{1}$. В приближении плавной неоднородности выражение для части свободной энергии, обусловленной неоднородностью распределения жидкости, может быть записано следующим образом:

$$
\delta \Phi=\frac{1}{2} \int_{R_{1}}^{R_{2}}\left(a(\delta \rho)^{2}+b(\nabla \delta \rho)^{2}+2 h(r) \delta \rho\right) r d r,
$$

где $\delta \rho(r)$ определяет отклонение плотности от среднего значения, $a$ и $b$ - феноменологические параметры модели, $h(r)$ - потенциал внешнего поля: в данном случае это результат совместного действия пристеночного потенциала каждого из цилиндров. Как отмечалось выше, выражение (1) является, фактически, общепринятым разложением до второго порядка свободной энергии по отклонению плотности и ее градиента при условии малости отклонений плотности от однородного распределения [5]-[7]. 
Задача состоит в поиске минимума функционала (1), однако при этом необходимо учесть дополнительное ограничение

$$
\int_{R_{1}}^{R_{2}} \delta \rho \cdot r d r=0
$$

которое является следствием постоянства массы жидкости в системе при перераспределении ее плотности. Таким образом, необходимо решить изопериметрическую задачу, которая сводится к решению уравнения

$$
b \Delta \delta \rho-a \delta \rho=h(r)+\lambda
$$

с граничными условиями (возникающими при решении задачи минимизации функционала)

$$
\left.\frac{d \delta \rho}{d r}\right|_{r=R_{1}}=\left.\frac{d \delta \rho}{d r}\right|_{r=R_{2}}=0
$$

при дополнительном ограничении (2) - неизвестный параметр $\lambda$ ищется с учетом этого условия.

Несложно показать, что параметр $\lambda$ определяется соотношением

$$
\lambda=-\frac{1}{\pi\left(R_{2}^{2}-R_{1}^{2}\right)} \int_{R_{1}}^{R_{2}} h(r) r d r
$$

а общее решение может быть представлено в виде

$$
\delta \rho=\xi+\frac{1}{a \pi\left(R_{2}^{2}-R_{1}^{2}\right)} \int_{R_{1}}^{R_{2}} h(r) r d r
$$

где параметр $\xi$ удовлетворяет следующему уравнению и граничным условиям:

$$
b \Delta \xi-a \xi=h(r),\left.\quad \frac{d \xi}{d r}\right|_{r=R_{1}}=\left.\frac{d \xi}{d r}\right|_{r=R_{2}}=0 .
$$

Общее решение для параметра $\xi$ имеет вид

$$
\xi(r)=\sum_{n=1}^{\infty} \xi_{n} \phi_{n}(r)
$$

где

$$
\phi_{n}(r)=Y_{1}\left(\mu_{n}\right) J_{0}\left(\frac{\mu_{n} r}{R_{2}}\right)-J_{1}\left(\mu_{n}\right) Y_{0}\left(\frac{\mu_{n} r}{R_{2}}\right),
$$

$J_{k}(x)$ и $Y_{k}(x)$ - соответственно функции Бесселя первого и второго рода индексов $k=0,1$, параметры $\mu_{n}$ являются решениями уравнения

$$
J_{1}\left(\frac{\mu_{n} R_{1}}{R_{2}}\right) Y_{1}\left(\mu_{n}\right)=J_{1}\left(\mu_{n}\right) Y_{1}\left(\frac{\mu_{n} R_{1}}{R_{2}}\right) .
$$

Коэффициенты разложения (3) определяются как

$$
\xi_{n}=-\frac{h_{n}}{a+b\left(\mu_{n} / R_{2}\right)^{2}},
$$


где

$$
h_{n}=\frac{2 \pi^{2} \mu_{n}^{2}}{4 R_{2}^{2}-\left(\pi \mu_{n} R_{1} \phi_{n}\left(R_{1}\right)\right)^{2}} \int_{R_{1}}^{R_{2}} h(r) \phi_{n}(r) r d r .
$$

При $R_{1}=0$ получаем путем перехода к пределу условие $J_{1}\left(\mu_{n}\right)=0$ для вычисления параметров $\mu_{n}$. Выражения для собственных функций разложения и коэффициенты разложения для поля в этом случае переходят в следующие:

$$
\phi_{n}(r)=Y_{1}\left(\mu_{n}\right) J_{0}\left(\frac{\mu_{n} r}{R_{2}}\right), \quad h_{n}=\frac{\pi^{2} \mu_{n}^{2}}{2 R_{2}^{2}} \int_{0}^{R_{2}} h(r) \phi_{n}(r) r d r .
$$

Полученными результатами мы воспользуемся для построения решения для системы, состоящей из нескольких коаксиальных цилиндров с тонкими наноразмерными стенками.

\section{3. СИСТЕМА С НАНОРАЗМЕРНЫМИ СТЕНКАМИ И ЭФФЕКТИВНЫЙ ПРИСТЕНОЧНЫЙ ПОТЕНЦИАЛ}

Рассмотрим систему, состоящую из $N+1$ вложенных друг в друга коаксиальных цилиндров с радиусами $R_{k}=(k+1) R$, где $k=0,1, \ldots, N$. Для решения задачи необходимо знать пристеночный потенциал для каждого из вложенных цилиндров, причем с разных сторон цилиндра этот потенциал может быть различным. Для конкретности будем рассматривать потенциал вида "ступенька": пристеночный потенциал действует только на расстоянии $D \ll R$ от стенки цилиндра, величина потенциала постоянна и одинакова для каждой из ограничивающих прослойку поверхностей, но разная для разных прослоек. Хотя такой модельный потенциал является достаточно сильным упрощением, для систем с короткодействующими взаимодействиями (которые здесь рассматриваются) такое упрощение представляется вполне приемлемым. Будем предполагать, что в центральной цилиндрической области величина пристеночного потенциала известна и равна $U_{0}$. Величина пристеночного потенциала для слоя, размещенного между $k$-м и $(k+1)$-м цилиндрами, равна $U_{k}$ и определяется из условия непрерывности профиля распределения плотности.

На основе полученного выше решения для коэффициентов разложения внешнего поля в ряд по функциям

$$
\phi_{n}^{(k)}(r)=Y_{1}\left(\mu_{n}\right) J_{0}\left(\frac{\mu_{n}^{(k)} r}{(k+1) R}\right)-J_{1}\left(\mu_{n}\right) Y_{0}\left(\frac{\mu_{n}^{(k)} r}{(k+1) R}\right)
$$

для $k$-го слоя $(k R \leqslant r \leqslant(k+1) R)$ получаем

$$
\begin{aligned}
h_{n}^{(k)}= & \frac{2 \pi^{2}\left(\mu_{n}^{(k)}\right)^{2}}{4(k+1)^{2} R^{2}-\left(\pi \mu_{n}^{(k)} k R \phi_{n}^{(k)}(k R)\right)^{2}} \times \\
& \times\left(\int_{k R}^{k R+D} h(r) \phi_{n}^{(k)}(r) r d r+\int_{(k+1) R-D}^{(k+1) R} h(r) \phi_{n}^{(k)}(r) r d r\right) \approx \\
\approx & \frac{2 \pi^{2}\left(\mu_{n}^{(k)}\right)^{2} D U_{k}\left[\phi_{n}^{(k)}(k R)+\phi_{n}^{(k)}((k+1) R)\right]}{4(k+1)^{2} R^{2}-\left(\pi \mu_{n}^{(k)} k R \phi_{n}^{(k)}(k R)\right)^{2}} .
\end{aligned}
$$


Собственные числа $\mu_{n}^{(k)}$ при $k>0$ определяются из соотношения

$$
J_{1}\left(\frac{\mu_{n}^{(k)} k}{k+1}\right) Y_{1}\left(\mu_{n}^{(k)}\right)=J_{1}\left(\mu_{n}^{(k)}\right) Y_{1}\left(\frac{\mu_{n}^{(k)} k}{k+1}\right) .
$$

При $k=0$, т.е. для центрального цилиндра, имеем

$$
h_{n}^{(0)}=\frac{\pi^{2}\left(\mu_{n}^{(0)}\right)^{2} U_{0}}{2 R^{2}} \int_{R-D}^{R} h(r) \phi_{n}^{(0)}(r) r d r \approx \frac{\pi^{2}\left(\mu_{n}^{(0)}\right)^{2} U_{0} \phi_{n}^{(0)}(R) D}{2 R^{2}},
$$

где

$$
\phi_{n}^{(0)}(r)=Y_{1}\left(\mu_{n}\right) J_{0}\left(\frac{\mu_{n}^{(0)} r}{R}\right)
$$

а $\mu_{n}^{(0)}$ удовлетворяет условию $J_{0}\left(\mu_{n}^{(0)}\right)=0$.

Для слоя с номером $k>0$ общее решение для распределения плотности дается выражением

$$
\begin{aligned}
\delta \rho(r)= & \frac{2 U_{k} D}{a \pi(2 k+1) R^{2}}- \\
& \quad-\pi^{2} D U_{k} \sum_{n=1}^{\infty} \frac{\left(\mu_{n}^{(k)}\right)^{2}\left[\phi_{n}^{(k)}(k R)+\phi_{n}^{(k)}((k+1) R)\right] \phi_{n}^{(k)}(r)}{\left[4(k+1)^{2}-\left(\pi \mu_{n}^{(k)} k \phi_{n}^{(k)}(k R)\right)^{2}\right]\left[a R^{2}+b\left(\mu_{n}^{(k)}\right)^{2}\right]},
\end{aligned}
$$

где $k R \leqslant r \leqslant(k+1) R$. Для случая $k=0$ (центральный цилиндр) решение имеет вид

$$
\delta \rho(r)=\frac{U_{0} D}{a \pi R^{2}}-\frac{\pi^{2} D U_{0}}{2} \sum_{n=1}^{\infty} \frac{\left(\mu_{n}^{(0)}\right)^{2} \phi_{n}^{(0)}(R) \phi_{n}^{(0)}(r)}{a R^{2}+b\left(\mu_{n}^{(0)}\right)^{2}},
$$

где $0 \leqslant r \leqslant R$. Если ввести обозначения

$$
\psi_{k}(r)=\frac{2}{a \pi(2 k+1)}-2 \pi^{2} \sum_{n=1}^{\infty} \frac{\left(\mu_{n}^{(k)}\right)^{2}\left[\phi_{n}^{(k)}(k R)+\phi_{n}^{(k)}((k+1) R)\right] \phi_{n}^{(k)}(r)}{\left[4(k+1)^{2}-\left(\pi \mu_{n}^{(k)} k \phi_{n}^{(k)}(k R)\right)^{2}\right]\left[a+b\left(\mu_{n}^{(k)} / R\right)^{2}\right]}
$$

при $k>0$ и

$$
\psi_{0}(r)=\frac{1}{a \pi}-\frac{\pi^{2}}{2} \sum_{n=1}^{\infty} \frac{\left(\mu_{n}^{(0)}\right)^{2} \phi_{n}^{(0)}(R) \phi_{n}^{(0)}(r)}{a+b\left(\mu_{n}^{(0)} / R\right)^{2}}
$$

для $k=0$, то мы можем записать для потенциала $n$-го слоя $(1 \leqslant n \leqslant N)$ следующее выражение:

$$
U_{n}=U_{0} \prod_{k=1}^{n} \frac{\psi_{k-1}((k+1) R)}{\psi_{k}((k+1) R)},
$$

где функции $\psi_{k}(r)$ определяются соотношениями (4) и (5). Данное выражение позволяет на феноменологическом уровне описать эффективный пристеночный потенциал рассматриваемой системы, что особенно важно при прогнозировании свойств пространственно ограниченных жидкостей означенной геометрии. Содержащиеся в этом выражении неизвестные параметры (такие, например, как значение $U_{0}$ ) могут быть оценены на основе экспериментальных данных. 


\section{4. ЗАКЛЮЧЕНИЕ}

Полученное общее решение для распределения плотности в пространственно ограниченной жидкой системе с геометрией межцилиндрической прослойки представляет определенный практический интерес как отдельная задача, поскольку открывает перспективы эффективной обработки и анализа экспериментальных данных по рассеянию нейтронов и критической опалесценции света в системах с такой геометрией. Что касается оценок эффективного пристеночного потенциала, то они, хотя и являются достаточно упрощенными, могут представлять интерес для экспериментаторов. Дело в том, что значительное количество расчетов в теории жидкостей базируется на использовании явных выражений для потенциалов прямого взаимодействия (частиц в жидкости или частиц с ограничивающими поверхностями). Поскольку точное выражение для потенциалов установить невозможно в принципе, обычно используются модельные потенциалы (см., например, книги [2], [8]). При этом важно иметь возможность определять параметры, входящие в соответствующие выражения для модельных потенциалов, и соотносить их с экспериментальными данными. Смеем надеяться, что представленные выше результаты позволяют сделать шаг в данном направлении.

\section{Список литературы}

[1] Л.А. Булавин, Нейтроннъе исследования динамики жидкостей, Вища школа, К., 1977.

[2] В. Я. Антонченко, А. С. Давыдов, В. В. Ильин, Основы физики водъ, Наукова думка, K., 1991.

[3] И. М. Франк, УФН, 161:11 (1991), 109-127.

[4] I. Brovchenko, A. Geiger, A. Oleinikova, D. Paschek, Eur. Phys. J. E, 12:1 (2003), 69-76.

[5] Л. А. Булавин, Д. А. Гаврюшенко, В. М. Сысоев, ЖФХ, 70:3 (1996), 559-561; 70:8, 1525-1526; 70:11, 2102-2103.

[6] L. A. Bulavin, D. A. Gavryushenko, V. M. Sysoev, Ukr. J. Phys., 52:10 (2007), 934-938.

[7] А.Н. Васильев, ТМФ, 151:1 (2007), 149-154.

[8] К. Крокстон, Физика жидкого состояния, Мир, М., 1978.

Поступила в редакцию 2.09.2009

после доработки 6.10.2009 